Technological University Dublin ARROW@TU Dublin

Articles

2019-9

\title{
Association of Total Zinc Intake with Myopia in U.S. Children and Adolescents
}

\author{
Niamh Burke \\ Technological University Dublin \\ John Butler \\ Technological University Dublin, john.s.butler@tudublin.ie \\ Ian Flitcroft \\ Technological University Dublin
}

See next page for additional authors

Follow this and additional works at: https://arrow.tudublin.ie/otpomart

Part of the Optometry Commons

\section{Recommended Citation}

Burke N, et al.(2019) Association of Total Zinc Intake with Myopia in US Children and Adolescents. Optometry and Vision Science. 2019;96:647-54. doi:10.1097/OPX.0000000000001418

This Article is brought to you for free and open access by ARROW@TU Dublin. It has been accepted for inclusion in Articles by an authorized administrator of ARROW@TU

Dublin. For more information, please contact arrow.admin@tudublin.ie, aisling.coyne@tudublin.ie, gerard.connolly@tudublin.ie.

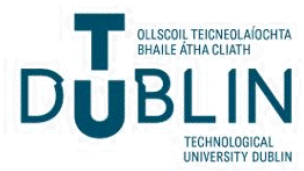




\section{Authors}

Niamh Burke, John Butler, lan Flitcroft, Daniel McCartney, and James Loughman 


\section{ARTICLE COVERSHEET}

\section{LWW_CONDENSED(8.13X10.88) \\ SERVER-BASED}

Article : OPX50777

Manuscript Number : OVS18407

Creator : dpc_Iww

Date : Tuesday July 30th 2019

Time : 14:02:07

Number of Pages (including this page) : 10 


\section{Association of Total Zinc Intake with Myopia in U.S. Children and Adolescents}

AQ1 Niamh Burke, MSc, ${ }^{1 *}$ John S. Butler, PhD, ${ }^{1,2}$ lan Flitcroft, FRCOphth, ${ }^{1,3}$ Daniel McCartney, PhD, ${ }^{1,4}$ and James Loughman, PhD ${ }^{1,5}$

SIGNIFICANCE: This present study advances our knowledge on the role of lifestyle factors in myopia (short-sightedness), specifically dietary factors. It has been suggested in previous studies that lower zinc status is associated with myopia; however, this article shows no relationship between dietary zinc intake and myopia in U.S. adolescents.

PURPOSE: It has been suggested that low zinc levels may contribute to the development of myopia. The aim of the present study is to examine, for the first time in a Western population, the association of total dietary and supplement zinc intake with myopia.

METHODS: A total of 1095 children/adolescents aged 12 to 19 years who participated in the U.S. National Health AQ2 and Nutrition Examination Survey from 2007 to 2008 were enrolled in this study. Multivariate logistic regression analysis was performed to examine the relationship between total zinc intake and myopia after adjustment for potential confounders. In addition, the association between total zinc intake and spherical equivalent refractive error was examined in the myopia group through multiple linear regression.

RESULTS: Among study participants, $30 \%$ were found to be myopic ( $\leq-1.00 \mathrm{D})$. Although median total daily zinc intake was lower among myopes (10.8 [10.2] mg/d) than among nonmyopes (11.1 [10.8] mg/d), the difference was not statistically significant $(P=.11)$. In multiple logistic regression analyses, zinc and copper intakes were not significantly associated with myopia after adjustment for age, sex, body mass index, ethnicity, family income, recreational activity, copper intake, and daily energy intake (in kilocalories per day). In multiple linear regression, spherical equivalent refractive error was not associated with total zinc intake in the myopic group after adjustment for confounding factors $(P=.13)$.

CONCLUSIONS: In contrast to previous Asian studies, total zinc intake is not associated with the presence of myopia in U.S. adolescents/children.

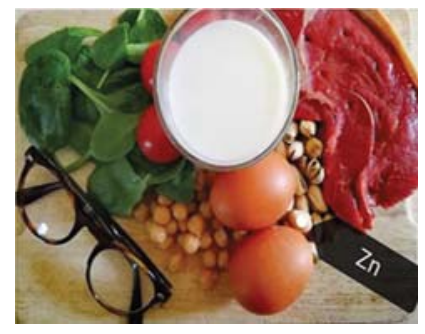

Author Affiliations:

${ }^{1}$ Centre for Eye Research Ireland, Environmental Sustainability and Health Institute, Dublin Institute of Technology, Grangegorman, Dublin, Ireland

${ }^{2}$ School of Mathematical Sciences, College of Sciences and Health, Institute of Techology, Dublin, Ireland

${ }^{3}$ Temple Street Children's University Hospital, Dublin, Ireland

${ }^{4}$ School of Biological and Health Sciences, College of Sciences and Health, of Fechology, Dublin, Ireland

${ }^{5}$ African Vision Research Institute, University of KwaZulu Natal, Durban, South Africa

*nia_burke@yahoo.com
The prevalence of myopia is increasing globally and has reached pandemic levels. Initial estimates, based on current data and comparable United Nations population statistics, predict that myopia will affect $50 \%$ (4949 million) of the world's population by 2050. ${ }^{1}$ The prevalence of myopia is highest in Asia Pacific and East Asia, with close to half of the population affected on average across both regions ${ }^{1}$ and almost universal prevalence among certain younger age groups (e.g., 96.5\% prevalence recently observed in young male individuals in the Republic of Korea). ${ }^{2}$ Prevalence levels are lower in Australia, Europe, the Americas, and other parts of Asia but are on the rise globally. ${ }^{1,3}$ Once thought of as an optical inconvenience, myopia is now accepted as a risk factor for potentially blinding ocular pathologies: cataract, glaucoma, retinal detachment, and myopic macular degeneration. ${ }^{4}$ It has become an economic and social burden for the state and individual, with very few successful therapeutic approaches to prevention or control.

The etiology of myopia is complex and unclear, and of late, there has been a significant increase in interest to identify causative factors. Studies have suggested several optical and environmental factors, ${ }^{5}$ which may contribute to its onset and progression including genetics, ${ }^{6}$ intensive near work, ${ }^{7}$ less time spent outdoors, ${ }^{8}$ and a signaling mechanism thought to involve peripheral hyperopic defocus as a component of a molecular feedback loop. ${ }^{9-11}$ It is likely, however, that a combination of influences is responsible for determining refractive status and not one factor acting in isolation.

Diet is an important environmental and lifestyle factor that has been studied in many eye diseases. Myopia in its early stages is very closely associated with growth, ${ }^{12}$ so it would seem plausible that deficits in nutritional intake and status could play a role in its development and progression. A number of observational studies have shown serum trace elements, such as zinc and copper, to be lower in myopic children/adolescents when compared with controls in parts of Asia. ${ }^{13,14}$ In addition, lower serum zinc levels have been linked with the pathological antecedents of myopia, thus suggesting that zinc may play a mechanistic role in myopia development. ${ }^{15,16}$ Physiologically, the highest concentration of zinc in the body is found in the eye, particularly the retina and choroid, and zinc deficiency has been shown in various studies to result in 
an array of structural and electrophysiological ocular manifestations. ${ }^{17}$ In fact, zinc is an integral element required for the structure and activity of many metalloenzymes and plays an important role in vitamin A metabolism. This role is potentially of interest in myopia given that retinoic acid, a metabolite of vitamin $A$, is thought to be an important molecular signal in regulating eye size and scleral extracellular matrix metabolism. ${ }^{18}$ Zinc is also a fundamental part of zinc finger DNA-binding proteins and assists in transcriptional processes and gene expression, in addition to having an important antioxidant role. Hence, there are numerous pathways by which zinc deficiency could contribute to the development and progression of myopia. ${ }^{19}$

Although there is preliminary evidence to support a relationship between zinc and myopia, those findings are specific to Asia and cannot be readily extrapolated to other populations for numerous reasons. Dietary habits, for example, are subject to regional variation due to cultural and agricultural/ecological differences. Such variation is likely to create differences in zinc intake and bioavailability across regions. ${ }^{20,21}$ In addition, the prevalence of myopia is much higher in Asia than in other regions, ${ }^{1}$ suggesting the presence of ethnic or constitutional susceptibilities, which may not prevail in other regions. There is therefore a need for similar investigations in a Western population.

There are currently no population-based studies of which we are aware that have specifically explored or reported on the relationship between dietary zinc intake and myopia. The National Health and Nutrition Examination Study is a program of studies designed to assess the health and nutritional status of adults and children in the United States. In the National Health and Nutrition Examination Study 2007 to 2008 cycle, data relating to dietary and supplement-based zinc intake and data on refractive error were simultaneously collected (refractive data have not been collected in more recent cycles). The aim of this study therefore is to test the hypothesis that low zinc intake may be associated with myopia in the U.S. population using a subsample of children/adolescents from the National Health and Nutrition Examination Study 2007 to 2008 .

\section{METHODS}

\section{Data Collection and Study Population}

A nationally representative sample of noninstitutionalized U.S. civilians was selected by using a complex, stratified, multistage sample design. The National Health and Nutrition Examination Study is an ongoing 2-year cycle of studies, in which data are collected by household interview or physical examination in mobile examination centers. The studies are administered by the National Center for Health Statistics at the Centers for Disease Control and Prevention. The study protocol was approved by the National Center for Health Statistics Institutional Review Board and adhered to the tenets of the Declaration of Helsinki. Informed consent was obtained from all participants. Additional information on the study design and methods is provided elsewhere. ${ }^{22}$

Data from the National Health and Nutrition Examination Study cycle 2007 to 2008, the most recent cycle with concurrent measures of vision and dietary zinc, were used in this analysis. In total, 10,149 individuals participated in the National Health and Nutrition Examination Study 2007 to 2008. Among those, all participants aged 12 to 19 years $(n=1156)$ were selected for this study. This age range was selected to prioritize the analysis of dietary intake during school-going years, the period during which myopia most frequently develops. Of these 1156 participants, 61 had missing refractive error data and were excluded. No reason was provided for the missing data. Analysis of the demographic profile of the participants with missing refractive error data reveals that the missing data seem to be randomly spread across sex, socioeconomic, and ethnic groups. In addition, participants with severe eye infection or incomplete, missing, or unreliable data for 24-hour recall nutrient intake were excluded (Fig. 1). Finally, 1095 participants were F1 found to be eligible for inclusion in the analysis.

\section{Participant Data and Measurements}

All participants underwent automated objective refraction/ keratometric evaluation on both eyes, carried out by trained health technicians. Participants' refractive error and corneal curvature were measured three times, without cycloplegia, using the Nidek AQ3 Autorefractor Model ARK-760 instrument. Refraction measurements were converted into spherical equivalent refraction, calculated as the spherical value plus half of the astigmatic value. Myopia was defined by spherical equivalent refraction of $\leq-1.00 \mathrm{D}$ because of the absence of cycloplegia in the method. ${ }^{23}$

Demographic information including age, sex, ethnicity (Mexican American, other Hispanic, non-Hispanic white, non-Hispanic black, other race), and family income were obtained from in-person household interviews. Body measurements such as height (in centimeters), weight (in kilograms), and waist circumference (in centimeters) were taken during physical examinations in the mobile examination center. Body mass index was calculated as weight (in kilograms) divided by height (in meters) squared. Physical activity levels were categorized using the Global Physical Activity Questionnaire. ${ }^{24}$ Moderate physical activity was categorized as "yes" if participants engaged in any moderate-intensity sports, fitness, or recreational activities that cause a small increase in breathing or heart rate for at least 10 minutes continuously, such as brisk walking, cycling, and swimming.

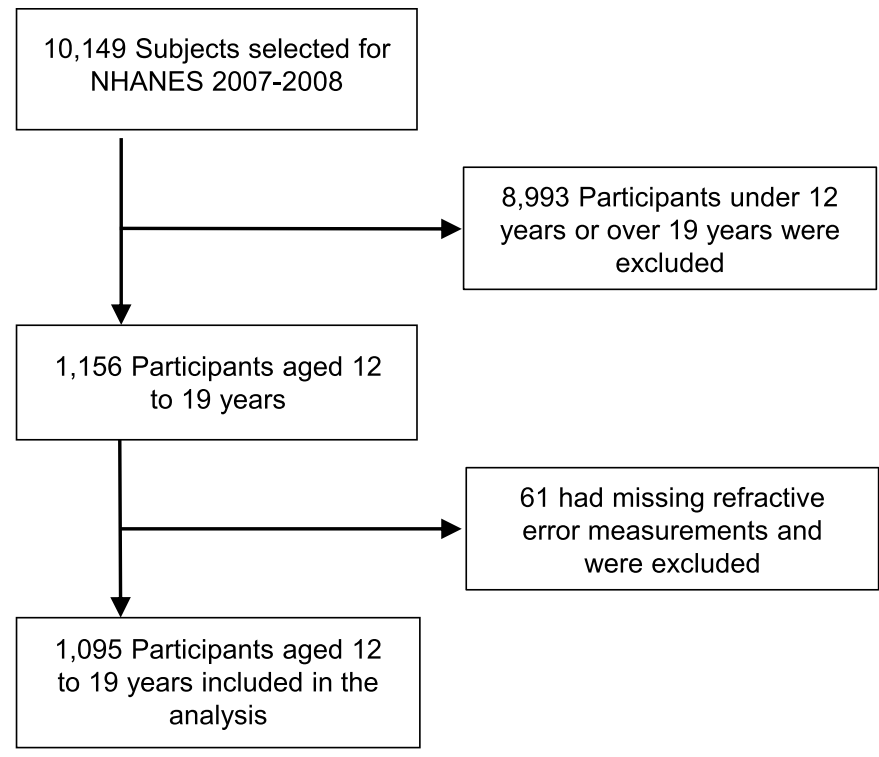

FIGURE 1. Flow diagram presenting the selection of study participants. 


\section{Dietary and Supplemental Intake Assessment}

Dietary intake was assessed using two 24-hour dietary recall interviews. Dietary intake data were used to estimate the types and amounts of foods and beverages (including all types of water) consumed during the 24-hour period before the interview (midnight to midnight) and to estimate intakes of energy, nutrients, and other food components from those food and beverages. ${ }^{25}$ The first dietary interview was administered in a private setting in the mobile examination center, and the second interview was conducted by telephone over the following 3 to 10 days. During the interviews, a multiple-pass protocol was used. Interviewers used a predefined script incorporated into the dietary recall system to ensure consistency in administration of the interviews. Nutrient intakes were calculated using the U.S. Department of Agriculture Food and Nutrient Databases for dietary analysis. In this study, these dietary recall data were used to calculate total daily energy intake and total

AQ4 nutrient intake of zinc and copper from food and beverages. Copper intake was included because of the known antagonistic effect of zinc intake on copper absorption. ${ }^{26}$ The 24 -hour dietary supplement questionnaire was administered after the 24-hour dietary recall using a similar protocol. Information on all vitamins, minerals, herbals, and nonprescription antacids that were consumed during a 24-hour period was obtained. Total nutrient intake from food and beverages was summed with total intake from supplements to calculate total nutrient intake of zinc and copper.

\section{Statistical Analysis}

All statistical analyses were performed using SAS survey procedure (version 9.4; SAS Institute, Inc., Cary, NC) to reflect the complex sampling design and sampling weights of the National Health and Nutrition Examination Study and to provide nationally representative prevalence estimates. Participants' characteristics were described using means and standard errors for continuous variables, and numbers and percentages for categorical variables according to spherical equivalent. For analyses of continuous and categorical variables, $t$ tests and design-adjusted Rao-Scott Pearson-type $x^{2}$ tests, respectively, were used. Total nutrient intakes were presented as median with interquartile range.

To estimate the relationship between total zinc and total copper intake with the health outcome of myopia, the National Cancer Institute method was used. This method was first used to estimate usual intake of zinc and copper from 24-hour dietary recall interviews. ${ }^{27}$ The two primary challenges of dietary data are measurement error adjustment and positively skewed data. Regression calibration and Box-Cox transformation were used to overcome these (the National Cancer Institute method uses adaptive Gaussian quadrate), and 24-hour recall data were replaced with a predictor of true intake of total zinc and total copper using 24-hour recall data and age and sex as covariates to aid estimates. This predictor of usual intake was then used to analyze zinc and copper as a potential independent variable of myopia in the health outcome model. This mixed-effects method was implemented using SAS macros MIXTRAN, DISTRIB, and INDIVINT V1.1. These macros were downloaded at https://epi.grants.cancer.gov/diet/ usualintakes/macros_single.html\#ver1.

The usual intakes of nutrient zinc and copper were divided into quartiles and treated as an explanatory variable for myopia in simple and multiple logistic regression, with quartile 1 as the reference category. Model 1 was adjusted for age and sex. Model 2 was adjusted for age, sex, ethnicity, family income, body mass index, recreational activity, copper, and total energy intake (in kilocalories per day). Tests for linear trends were conducted using regression with a median value of each category of the analyzed exposure as a single ordinal variable. To further explore the relationship between usual intake of zinc and myopia, we performed multivariate linear regression on the association between total zinc intake and spherical equivalent refractive error in the myopic population. All reported probabilities were two sided, with $P<.05$ considered statistically significant.

\section{RESULTS}

The demographic, anthropometric, and dietary intake characteristics of study participants according to refractive status are reported in Table 1. Of the 1095 participants, 30\% were myopic T1 $(\leq-1.00 \mathrm{D})$. Spherical equivalent refractive error in the right and left eyes was highly correlated (Pearson correlation $=0.87, P<$ .001). Therefore, only data for the right eyes are presented.

As expected, there was a significant difference in spherical equivalent between the myopic and nonmyopic participant groups $(P<.001)$. There were, however, no significant differences in demographic (sex, age, ethnicity, family income) or anthropometric (height, weight, and waist circumference) characteristics between myopic and nonmyopic participants $(P>.05$ for all). Similarly no significant difference was found in body mass index or frequency of regular physical activity between myopes and nonmyopes; however, $P$ values of .06 and .06 , respectively, were noted.

The median daily total zinc, copper, and energy intakes of participants with myopia were not significantly different from those without myopia.

The association between total zinc and copper intakes and myopia in simple and multiple logistic regression models is presented in Table 2. The simple odds ratio with 95\% confidence intervals in- T2 dicated that total zinc and copper intakes were not associated with myopia. The lack of association persisted after initial adjustment for age and sex and after additional adjustment for body mass index, ethnicity, family income, recreational activity, copper, and total daily energy intake in multivariate analysis. The multivariate-adjusted odds ratios (95\% confidence intervals) for myopia were 0.88 (0.59 to $1.30 ; P=.57)$ and $1.19(0.68$ to $2.08 ; P=.18)$ in the highest versus lowest quartile of zinc and copper intakes, respectively. Similarly, the copper-to-zinc ratio was not associated with risk of myopia (odds ratio, 1.06 [0.65 to 1.71$] ; P=.29$ ).

The results of multiple linear regression are shown in Table 3. T3 Spherical equivalent refractive error was not significantly associated with total zinc intake in the myopic group in either the simple model, or after adjustment for age and sex in model $1(P=.14)$, or after subsequent adjustment for body mass index, ethnicity, family income, recreational activity, copper intake, and total daily energy intake in model $2(P=.13)$.

All analyses were repeated for data in the previous cycle, National Health and Nutrition Examination Study 2005 to 2006, to confirm our findings herein. Similar statistical analysis (as outlined previously) was performed on eligible subjects aged 12 to 19 years $(n=1936)$. The median (interquartile range) intakes of zinc were $10.26(8.98)$ $\mathrm{mg} / \mathrm{d}$ in nonmyopes and $10.67(8.95) \mathrm{mg} / \mathrm{d}$ in myopes $(P=.51$; supplement-based zinc intake data were not included in this earlier cycle because the collection method differed from that of 2007 to 2008). The multivariate-adjusted odds ratio (95\% confidence interval) 
AQ5

TAB Demographic, anthropometric, and dietary intake characteristics of study participants according to refractive status

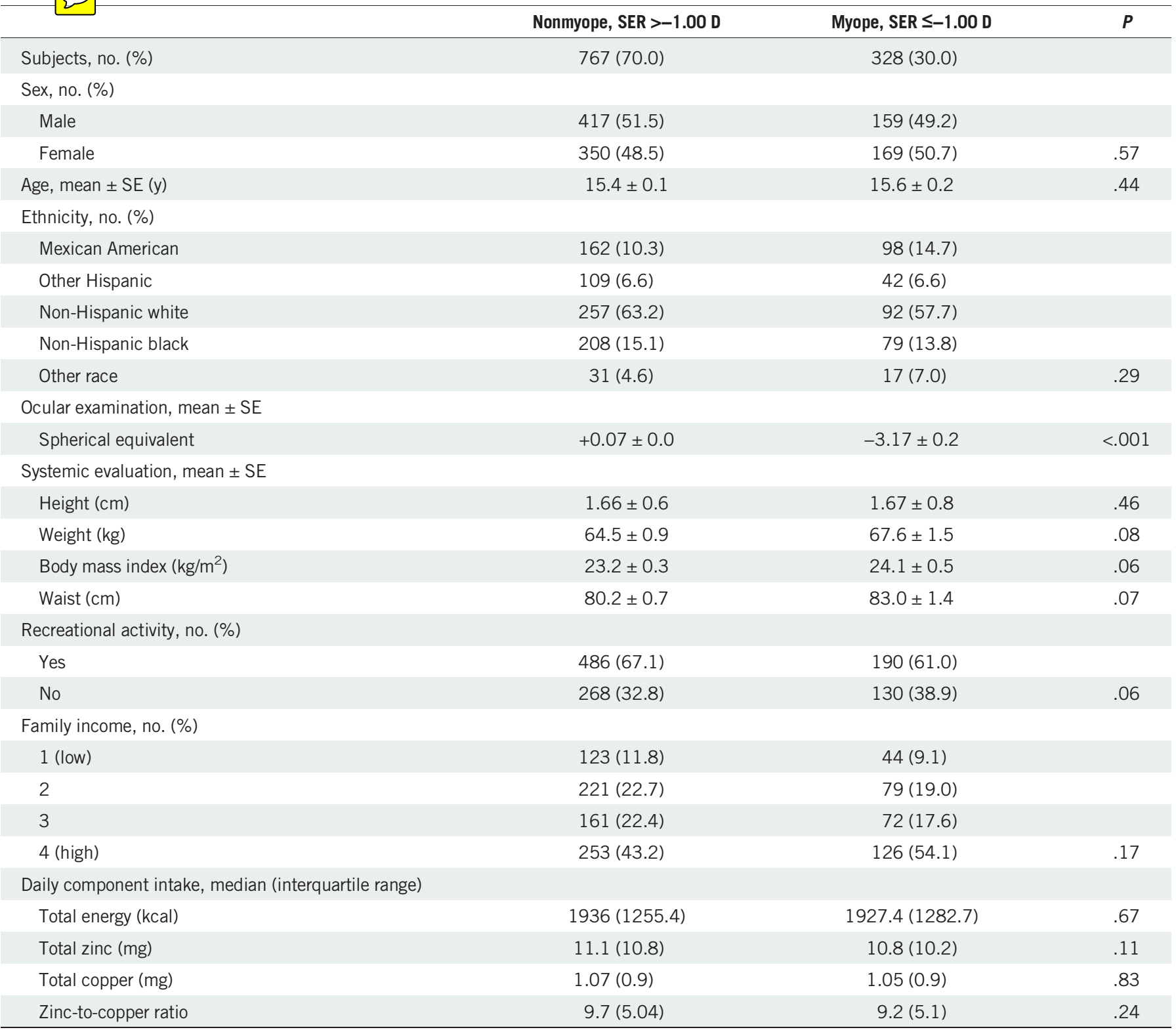

Data are presented as mean $\pm \mathrm{SE}$, number (percent), or median (interquartile range). SER $=$ spherical equivalent refractive error.

for myopia was $0.75(0.44$ to $1.24 ; P=.56)$ in the highest versus lowest quartile of zinc intake.

\section{DISCUSSION}

We found no significant association between dietary zinc intake and myopia in U.S. children/adolescents aged 12 to 19 years after adjustment for potential confounders. To our knowledge, this is the first study to explore the association between total zinc intake and myopia in children/adolescents using nationally representative data.

Our findings differ from those of previously published observational studies that have suggested that there may be an association between low zinc intake and status and myopia. A recent study by Fedor et al. ${ }^{15}$ on participants aged 7 to 17 years found the average serum zinc concentration in patients with myopia $(0.865 \pm 0.221 \mathrm{mg} / \mathrm{L})$ to be significantly lower than that in controls $(1.054 \pm 0.174 \mathrm{mg} / \mathrm{L})$. In contrast to our study, a significantly higher copper/zinc ratio was also found in myopic participants compared with controls $(1.196 \pm 0.45$ vs. $0.992 \pm 0.203)$, indicating possible disruption of antioxidant defenses. The sample size was limited $(n=121)$, however, and only moderate to high myopes were included in the study (spherical equivalent, $\leq-3 \mathrm{D}$ ), with the myopic group having a particularly high mean spherical equivalent of $-7.31 \mathrm{D} .{ }^{15}$

An examination of serum micronutrient levels (zinc, iron, copper, magnesium, manganese, and lead) involving 120 myopic primary school students was also carried out in Dongguan, China. Serum levels of zinc and iron were found to be significantly lower in myopes than in controls, whereas there was no significant difference in other trace elements measured. ${ }^{13}$ 
TABLE 2. Weighted odds ratio (95\% confidence intervals) of myopia across quartiles of zinc intake, NHANES 2007 to 2008

\begin{tabular}{|c|c|c|c|c|}
\hline & Intake cutoff & Simple & Model 1 & Model 2 \\
\hline \multicolumn{5}{|c|}{ Total zinc intake (mg/d) } \\
\hline Quartile 1 (low) & $<8.8$ & 1 (ref) & 1 (ref) & 1 (ref) \\
\hline Quartile 3 & 11.4 to $<14.2$ & $0.71(0.39-1.29)$ & $0.72(0.38-1.35)$ & $0.69(0.37-1.30)$ \\
\hline Quartile 4 (high) & $\geq 14.2$ & $0.85(0.60-1.21)$ & $0.86(0.59-1.30)$ & $0.88(0.59-1.3)$ \\
\hline \multicolumn{5}{|c|}{ Total copper intake (mg/d) } \\
\hline Quartile 1 (low) & $<0.90$ & 1 (ref) & 1 (ref) & 1 (ref) \\
\hline Quartile 2 & 0.90 to $<1.12$ & $0.66(0.37-1.17)$ & $0.66(0.37-1.17)$ & $0.67(0.35-1.32)$ \\
\hline Quartile 3 & 1.12 to $<1.38$ & $0.97(0.67-1.42)$ & $0.98(0.67-1.43)$ & $1.12(0.75-1.76)$ \\
\hline Quartile 4 (high) & $\geq 1.38$ & $1.00(0.67-1.48)$ & $1.00(0.65-1.56)$ & $1.19(0.68-2.08)$ \\
\hline \multicolumn{5}{|l|}{ Zinc-to-copper ratio } \\
\hline Quartile 2 & 7.37 to $<9.62$ & $1.00(0.75-1.33)$ & $0.98(0.74-1.31)$ & $1.04(0.95-1.39)$ \\
\hline Quartile 3 & 9.62 to $<12.47$ & $0.73(0.52-1.03)$ & $1.36(0.97-1.90)$ & $1.34(0.95-1.90)$ \\
\hline Quartile 4 & $\geq 12.47$ & $0.96(0.62-1.49)$ & $1.03(0.66-1.60)$ & $1.06(0.65-1.71)$ \\
\hline$P$ trend & & .35 & .36 & .29 \\
\hline
\end{tabular}

Model 1 adjusted for age and sex. Model 2 adjusted for copper intake, age, sex, ethnicity, body mass index, family income, recreational activity, and daily energy intake (in kilocalories per day, continuous). NHANES = National Health and Nutrition Examination Survey; ref = reference.

Another study investigating serum levels of zinc, copper, and selenium in 168 Chinese middle school students reported levels of all trace elements in the myopic group, which were much lower than the levels observed in the emmetropic group, whereas degree of myopia was negatively correlated with levels of serum zinc and copper. ${ }^{14}$ These findings were supported by Xie et al., ${ }^{16}$ who reported significantly lower zinc and iron concentrations and higher copper concentrations in the serum of their myopic patients. Zinc was also negatively correlated with the degree of myopia in this study. Interestingly, and important to note, although serum zinc levels were found to be lower in myopic children/adolescents, no study found myopes to have zinc deficiency levels $(<70 \mu \mathrm{g} / \mathrm{dL})$.

There are numerous possible explanations for the conflicting findings reported herein. Study design and methodological differences need to be acknowledged, as our study only measured dietary zinc intake, and recently published evidence on National Health and Nutrition Examination Study data suggests that serum zinc concentration in the U.S. population is not related to dietary or supplemental zinc. ${ }^{28}$ Population-based differences also merit consideration, as most previous studies were carried out on Asian populations; thus,

TABLE 3. Multiple linear regression analysis for the association between total zinc intake and SER in the myopic group $(n=328)$

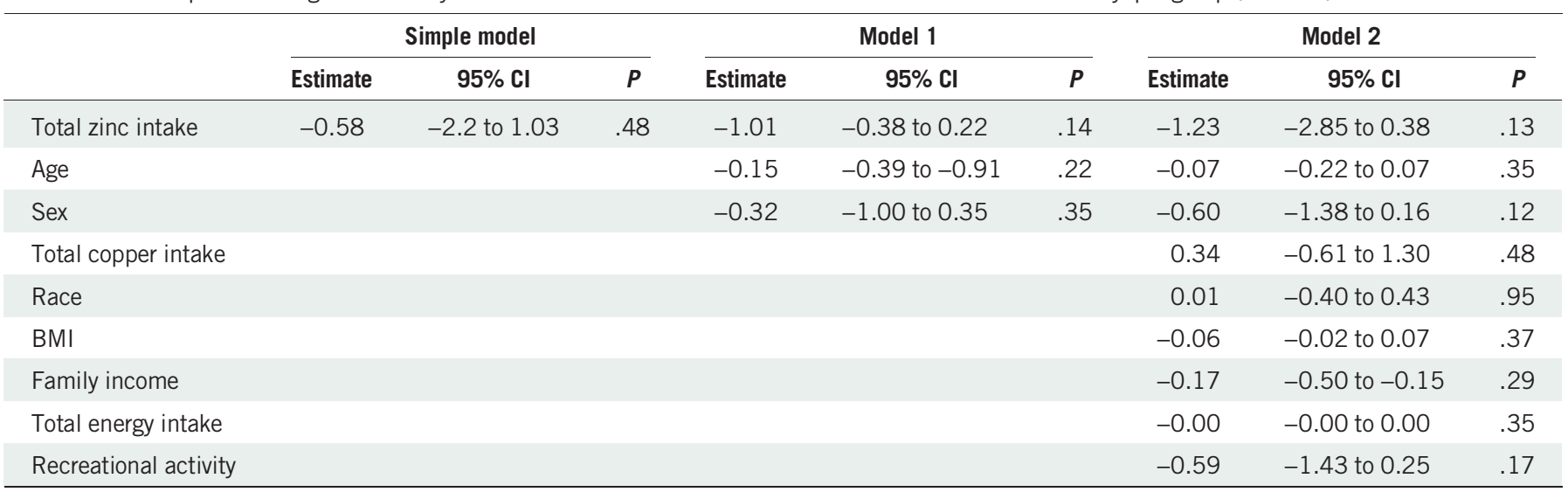

Model 1 adjusted for age and sex. Model 2 adjusted for copper intake, age, sex, ethnicity, body mass index, family income, recreational activity, and daily energy intake. $\mathrm{BMI}=$ body mass index; $\mathrm{Cl}=$ confidence interval; SER = spherical equivalent refractive error. 
genetic, lifestyle, or dietary differences may have contributed to the variation in results.

According to the World Health Organization, the estimated global prevalence of zinc deficiency is $31 \%$. Prevalence is lower in Europe and North America and higher in areas of the Eastern Mediterranean, South, and Southeast Asia, most likely due to dietary variance. ${ }^{29}$ Animal products such as red meat and dairy are major sources of dietary zinc in Western countries; diets based on cereals and legumes, which are lower in animal products, make it difficult to meet zinc requirements. Bioavailability of zinc is also lower in plant foods (cereals, grains, vegetables, and soybeans) because they are high in phytate, a known inhibitor of zinc absorption. ${ }^{30,31}$ Marginal deficiency and suboptimal zinc status are also prevalent but much more difficult to identify, and because of high demand for zinc and limitations in body stores, children are particularly vulnerable to the consequences of reduced zinc levels. ${ }^{30}$ The current recommended daily allowance for zinc intake in U.S. children is $8 \mathrm{mg}$ for both boys and girls, and those in adolescents are $11 \mathrm{mg}$ for boys and $9 \mathrm{mg}$ for girls. In this study, the lowest quartile of zinc intake was $<6.3 \mathrm{mg} / \mathrm{d}$ and included 226 participants, which means that a significant number of subjects had inadequate zinc intake.

A substantial number of proteins and enzymes contain zinc, which explains the importance of zinc in many cell processes. ${ }^{32}$ Zinc has several actions in many of these enzymes, such as catalytic, structural, substrate, or regulatory roles in enzyme activity. ${ }^{33}$ The extensive number of zinc-reliant enzymes explains zinc's necessity in DNA, RNA, and protein and lipid metabolism and synthesis. Zinc also plays an important role in maintaining the genome. ${ }^{34}$ This requires, among other factors, the antioxidant effects of zinc and its involvement in DNA repair in response to DNA damage and in the compositional makeup of biomolecules (e.g., methionine), which are required for DNA methylation. ${ }^{34}$ Zinc also regulates the storage and release of neurotransmitters, ${ }^{35}$ so it is clear that there are many potential pathological mechanisms by which zinc deficiency could play a role in myopia. Although no clear relationship between zinc intake and myopia was observed herein, there is sufficient rationale and serum evidence that some of the candidate pathways through which zinc may influence AQ4 myopia development are worth exploring. These could include disruption of vitamin A metabolism, impairment of antioxidant defenses, disruption of epigenetic regulators, and adverse effects on blood glucose control, among others.

As previously mentioned, retinoic acid, a metabolite of vita$\min A$, has been shown to be involved in the signaling pathway, which modulates eye growth between the retina and the sclera. ${ }^{36}$ The level of zinc in the body affects numerous aspects of vitamin A metabolism, namely, absorption, transport, and usage. ${ }^{37}$ Zinc is understood to control vitamin A transport by protein synthesis and oxidative conversion of retinol to retinal, a process that requires the action of zinc-dependent retinol dehydrogenase enzyme. Retinal is subsequently converted to retinoic acid by retinaldehyde dehydrogenases. ${ }^{37}$ In animal models, it has been shown that choroidal synthesis of all-trans retinoic acid alters during times of visually induced changes in ocular growth and that this has a distinct effect on scleral extracellular matrix metabolism. ${ }^{38,39}$ More research on these molecular processes and zinc's possible influence on changes in retinoic acid and scleral remodeling is required.

In recent years, some evidence has suggested oxidative stress as an important factor in altered pathways in myopia and the development of associated eye diseases. ${ }^{40}$ It is known that lack of zinc increases oxidative stress, resulting in oxidative damage to DNA, proteins, and lipids. ${ }^{41}$ Physiologically, zinc levels in the retina, choroid, sclera, and ciliary muscle are higher than those in other intraocular tissues. Zinc is capable of reducing oxidative stress by several mechanisms: activation of antioxidant proteins, molecules, and enzymes (metallothioneins, glutathione, superoxide dismutase); reduction of highly reactive hydroxyl radical; and the inhibition of activities of oxidant-promoting enzymes, such as inducible nitric oxide synthase and NADPH oxidase. ${ }^{40}$ In an animal model of myopia, Huibi et al. ${ }^{42}$ detected reduced superoxide dismutase, nitric oxide synthase activity, and nitric oxide content in the retinal pigment epithelium-choroid homogenate of chick eyes. Interestingly, after administration of the trace element zinc, these three parameters were increased, and axial elongation and myopia progression were inhibited.

It is thought that genes expressed in the retina, retinal pigment epithelial, choroid, and sclera, which are involved in the normal emmetropization process, could be involved in myopia development. Specifically, the irregular expression of these genes so that the emmetropization mechanism is disturbed may cause an increase in axial length and consequential development of myopia. ${ }^{43}$ Zinc has the ability to influence the expression of many genes, and it is part of the structure of more than 2500 transcription factors. ${ }^{44}$ Myopia development is thought to be influenced by environmental factors, and environmental factors can also influence gene expression through epigenetic changes. Several key enzymes and zinc finger proteins play an important role in epigenetics (DNA methylation and histone modification). ${ }^{45}$ A study by Zhou et al. ${ }^{46}$ investigated whether myopia development was associated with changes in scleral DNA methylation of collagen gene COL1A1 in chicks. This AQ6 group found that increased DNA methylation resulted in suppression of transcription of scleral COL1A1 (inhibiting scleral collagen synthesis) and suggested that low to moderate myopia might not be associated with DNA sequences alone but instead may relate to epigenetic changes in such regulatory genes.

In the human body, zinc is also highly concentrated in the pancreas, ${ }^{47}$ where it engages in several functions related to blood glucose control. Adequate dietary zinc intake has been associated with reduced fasting glucose levels. ${ }^{48}$ It has been hypothesized that overconsumption of foods with a high glycemic load, as part of the modern diet, results in an increase in circulating insulin levels, which aid unregulated scleral tissue growth through an increase in insulinlike growth factor 1 and depletion of the retinoic acid signal ${ }^{49}$ giving rise to myopia. If this hypothesis is correct, zinc deficiency or lower levels of zinc may contribute to the pathogenesis of myopia in this way or through a complex interplay of many but asyet uncertain mechanisms such as those highlighted here.

Although the strengths of this study include its large nationally representative sample, minimal selection bias, and independently collected data, this study does have several limitations. All National Health and Nutrition Examination Study dietary data are collected by 24-hour recall interview. All self-reported measures of dietary recall are subject to error; although the automated multiple-pass method of data collection mitigates this risk, recall may still be inaccurate or biased. ${ }^{50}$ Also, as discussed previously, phytate content of foods may affect zinc absorption, so dietary intake often does not reflect zinc status. In this context, a biological biomarker such as serum or hair zinc may be a better, more accurate parameter to assess any potential role of zinc on myopia or other ocular health outcomes. ${ }^{51}$ In this study, refractive status was not checked under cycloplegic conditions; thus, myopia status may have been overestimated because of involuntary accommodation. In addition, other variables such as recreational activity and family income 
were self-reported, leaving measurements open to bias. ${ }^{52,53}$ Also, this study might be confounded by lack of data on other relevant variables such as information on total time spent outdoors and parental myopic status.
Dietary zinc intake is not associated with myopia in a teenage U.S. population; however, further investigation is warranted using a biomarker of zinc status rather than relying exclusively on dietary intake as a proxy for zinc status.

\section{ARTICLE INFORMATION}

Submitted: December 20, 2018

Accepted: May 24, 2019

AQ7 Funding/Support: None of the authors have reported, funding/support.

Conflict of Interest Disclosure: None of the authors have reported a financial conflict of interest.

Author Contributions and Acknowledgments: Conceptualization: NB, IF, JL; Data Curation: NB; Formal Analysis: NB, JSB; Investigation: JSB, IF; Methodology: NB, JSB, DM, JL; Supervision: JSB, IF, JL; Writing - Original Draft: NB Writing - Review \& Editing: NB, JSB, IF, DM, JL.

We thank all individuals at the National Center for Health Statistics of the Centers for Disease Control and Prevention who were responsible for the planning and administrating of the National Health and Nutrition Examination Survey and making the data sets of the National Health and Nutrition Examination Survey available on their website.

\section{REFERENCES}

1. Holden BA, Fricke TR, Wilson DA, et al. Global Prevalence of Myopia and High Myopia and Temporal Trends from 2000 through 2050. Ophthalmology 2016;123: 1036-42.

2. Jung SK, Lee JH, Kakizaki H, et al. Prevalence of Myopia and Its Association with Body Stature and Educational Level in 19-year-old Male Conscripts in Seoul, South Korea. Invest Ophthalmol Vis Sci 2012;53: 5579-83.

3. Smith TS, Frick KD, Holden BA, et al. Potential Lost Productivity Resulting from the Global Burden of Uncorrected Refractive Error. Bull World Health Organ 2009; 87:431-7.

4. Wong TY, Ferreira A, Hughes R, et al. Epidemiology and Disease Burden of Pathologic Myopia and Myopic Choroidal Neovascularization: An Evidence-based Systematic Review. Am J Ophthalmol 2014;157: 9-25.e12.

5. Morgan IG, Ohno-Matsui K, Saw SM. Myopia. Lancet 2012;379:1739-48.

6. Mutti DO, Mitchell GL, Moeschberger ML, et al. Parental Myopia, Near Work, School Achievement, and Children's Refractive Error. Invest Ophthalmol Vis Sci 2002;43:3633-40.

7. Saw SM, Hong RZ, Zhang MZ, et al. Near-work Activity and Myopia in Rural and Urban Schoolchildren in China. J Pediatr Ophthalmol Strabismus 2001;38: 149-55.

8. Morgan IG, Xiang F, Zeng Y, et al. Increased Outdoor Time Reduces Incident Myopia-The Guangzhou Outdoor Activity Longitudinal Study. Invest Ophthalmol Vis Sci 2014;55:1272.

9. Berntsen DA, Barr CD, Mutti DO, et al. Periphera Defocus and Myopia Progression in Myopic Children Randomly Assigned to Wear Single Vision and Progressive
Addition Lenses. Invest Ophthalmol Vis Sci 2013;54: 5761-70.

10. Benavente-Pérez A, Nour A, Troilo D. Axial Eye Growth and Refractive Error Development Can Be Modified by Exposing the Peripheral Retina to Relative Myopic or Hyperopic Defocus. Invest Ophthalmol Vis Sci 2014;55:6765-73.

11. Smith EL, 3rd, Hung LF, Arumugam B. Visual Regulation of Refractive Development: Insights from Animal Studies. Eye 2014;28:180-8.

12. Rudnicka AR, Kapetanakis VV, Wathern AK, et al. Global Variations and Time Trends in the Prevalence of Childhood Myopia, a Systematic Review and Quantitative Meta-analysis: Implications for Aetiology and Early Prevention. Br J Ophthalmol 2016;100:882-90.

13. Wang L. Variation Analysis of Six Kinds of Common Micro-elements Contents of Blood in Myopic Primary School Students in Dongguan District. Cent Chin Med J 2009; 1:20-2.

14. Huo M, Liu H, Cao J. The Relationship between Serum Zinc, Copper, Selenium and the Visions of Middle School Students. Chin J Sch Health 2006;4: 318-9.

15. Fedor M, Socha K, Urban B, et al. Serum Concentration of Zinc, Copper, Selenium, Manganese, and Cu/Zn Ratio in Children and Adolescents with Myopia. Biol Trace Elem Res 2017;176:1-9.

16. Xie X, He H, Wang J, et al. Clinical Significance of Serum Trace Elements in Juvenile Patients with Myopia. J Huaihai Med 2003;4:279-80.

17. Karcioglu ZA. Zinc in the Eye. Surv Ophthalmol 1982;27:114-22.

18. Summers JA, Harper AR, Feasley CL, et al. Identification of Apolipoprotein A-I as a Retinoic Acid-binding Protein in the Eye. J Biol Chem 2016;291:18991-9005.

19. Prasad AS. Zinc Is an Antioxidant and Anti-inflammatory Agent: Its Role in Human Health. Front Nutr 2014; $1: 14$.

20. Wessells KR, Brown KH. Estimating the Global Prevalence of Zinc Deficiency: Results Based on Zinc Availability in National Food Supplies and the Prevalence of Stunting. PLoS One 2012; 7:e50568.

21. Lönnerdal B. Dietary Factors Influencing Zinc Absorption. J Nutr 2000;130(Suppl. 5S):1378S-83.

22. Zipf G, Chiappa M, Porter KS, et al. National Health and Nutrition Examination Survey: Plan and Operations, 1999-2010. Vital Health Stat 1 2013;1-37.

23. Vitale S, Sperduto RD, Ferris FL, 3rd. Increased Prevalence of Myopia in the United States between 1971-1972 and 1999-2004. Arch Ophthalmol 2009; 127:1632-9.

24. Cleland CL, Hunter RF, Kee F, et al. Validity of the Global Physical Activity Questionnaire (GPAQ) in Assessing Levels and Change in Moderate-vigorous Physical Activity and Sedentary Behaviour. BMC Public Health 2014;14:1255.

25. Prentice RL, Mossavar-Rahmani Y, Huang Y, et al. Evaluation and Comparison of Food Records, Recalls, and Frequencies for Energy and Protein Assessment by
Using Recovery Biomarkers. Am J Epidemiol 2011; 174:591-603.

26. Fischer PWF, Giroux A, L'Abbe MR. The Effect of Dietary Zinc on Intestinal Copper Absorption. Am J Clin Nutr 1981;34:1670-5

27. Herrick KA, Rossen LM, Parsons R, et al. Estimating Usual Dietary Intake from National Health and Nutrition Examination Survey Data Using the National Cancer Institute Method. Vital Health Stat 2 2018;1-63.

28. Hennigar S, Lieberman HR, Fulgoni V, et al. Serum Zinc Concentrations in the US Population Are Related to Sex, Age, and Time of Blood Draw But Not Dietary or Supplemental Zinc. J Nutr 2018;148:1341-51.

29. World Health Organisation (WHO). Zinc Deficiency. Available at: https://www.who.int/publications/cra/chapters/ volume1/0257-0280.pdf. Accessed September 9, 2018.

30. Roohani N, Hurrell R, Kelishadi R, et al. Zinc and Its Importance for Human Health: An Integrative Review. J Res Med Sci 2013;18:144-57.

31. Joung H, Nam G, Yoon S, et al. Bioavailable Zinc Intake of Korean Adults in Relation to the Phytate Content of Korean Foods. J Food Composit Anal 2004;17: 713-24.

32. Oteiza PI. Zinc and the Modulation of Redox Homeostasis. Free Radic Biol Med 2012;53:1748-59.

33. Andreini C, Bertini I. A Bioinformatics View of Zinc Enzymes. J Inorg Biochem 2012;111:150-6.

34. Sharif $R$, Thomas $P$, Zalewski $P$, et al. The Role of Zinc in Genomic Stability. Mutat Res 2012;733: 111-21.

35. Bitanihirwe BK, Cunningham MG. Zinc: The Brain's Dark Horse. Synapse 2009;63:1029-49.

36. Harper AR, Wang X, Moiseyev G, et al. Postnatal Chick Choroids Exhibit Increased Retinaldehyde Dehydrogenase Activity during Recovery from Form Deprivation Induced Myopia. Invest Ophthalmol Vis Sci 2016; 57:4886-97.

37. Christian P, West KP, Jr. Interactions between Zinc and Vitamin A: An Update. Am J Clin Nutr 1998; 68(Suppl. 2):435S-41.

38. Mertz JR, Wallman J. Choroidal Retinoic Acid Synthesis: A Possible Mediator between Refractive Error and Compensatory Eye Growth. Exp Eye Res 2000;70: 519-27.

39. Seko Y, Shimizu M, Tokoro T. Retinoic Acid Increases in the Retina of the Chick with Form Deprivation Myopia. Ophthalmic Res 1998;30:361-7.

40. Francisco BM, Salvador M, Amparo N. Oxidative Stress AQ8 in Myopia. Oxid Med Cell Longev 2015;2015:750637.

41. Powell SR. The Antioxidant Properties of Zinc. J Nutr 2000;130:1447S-54.

42. Huibi X, Kaixun H, Qiuhua G, et al. Prevention of Axial Elongation in Myopia by the Trace Element Zinc. Biol Trace Elem Res 2001;79:39-47.

43. Young TL. Molecular Genetics of Human Myopia: An Update. Optom Vis Sci 2009;86:E8-22.

44. Olza J, Aranceta-Bartrina J, González-Gross M, et al. Reported Dietary Intake and Food Sources of Zinc, 
Selenium, and Vitamins A, E and C in the Spanish Population: Findings from the ANIBES Study. Nutrients 2017;9:E697.

45. Moore LD, Le T, Fan G. DNA Methylation and Its Basic Function. Neuropsychopharmacology 2012;38: 23-38.

46. Zhou X, Ji F, An J, et al. Experimental Murine Myopia Induces Collagen Type la1 (COL1A1) DNA Methylation and Altered COL1A1 Messenger RNA Expression in Sclera. Mol Vis 2012;18:1312-24.

47. Kelleher SL, McCormick NH, Velasquez V, et al. Zinc in Specialized Secretory Tissues: Roles in the
Pancreas, Prostate, and Mammary Gland. Adv Nutr 2011;2:101-11.

48. Wang Y, Jia XF, Zhang B, et al. Dietary Zinc Intake and Its Association with Metabolic Syndrome Indicators among Chinese Adults: An Analysis of the China Nutritional Transition Cohort Survey 2015. Nutrients 2018;10.

49. Cordain L, Eaton SB, Brand Miller J, et al. An Evolutionary Analysis of the Aetiology and Pathogenesis of Juvenile-onset Myopia. Acta Ophthalmol Scand 2002; 80:125-35.

50. Ahluwalia N, Dwyer J, Terry A, et al. Update on NHANES Dietary Data: Focus on Collection, Release,
Analytical Considerations, and Uses to Inform Public Policy. Adv Nutr 2016;7:121-34.

51. Wieringa FT, Dijkhuizen MA, Fiorentino M, et al. Determination of Zinc Status in Humans: Which Indicator Should We Use? Nutrients 2015;7:3252-63.

52. Sternfeld B, Goldman-Rosas L. A Systematic Approach to Selecting an Appropriate Measure of Selfreported Physical Activity or Sedentary Behaviour. J Phys Act Health 2012;9(Suppl. 1):S19-28.

53. Moore JC, Stinson LL, Welnick EJ. Income Measurement Error in Surveys: A Review. J Off Stat 2000;16: 331-61. 


\section{AUTHOR QUERIES}

\section{AUTHOR PLEASE ANSWER ALL QUERIES}

AQ1 = Please check if authors name are correctly captured for given names (in red) and surnames (in blue) for indexing after publication.

$\mathrm{AQ} 2=$ Please check if correct as modified ${ }_{1}$

AQ3 = Please indicate the location of Nidek.

AQ4 = This sentence has been slightly modified for clarity. Please check that the meaning is still correct, and amend if necessary.

AQ5 = Please check if Table 1 has been presented correctly ${ }_{1}$

AQ6 = As per journal style, genes and mutations should be in italics (and proteins should be in roman). Those explicitly described in the text as such were italicized. Please check and make modifications as necessary.

AQ7 = Please review and confirm the accuracy of the Funding/Support, Conflict of Interest Disclosure, and Author Contribution sections.

AQ8 = Ref. 42 was a duplicate and was thus removed from the Reference list. Please check if appropriate.

END OF AUTHOR QUERIES 